\title{
MITOTIC CYCLES IN TRITICUM SPECIES
}

\author{
P. O. L. DAVIES and H. REES \\ Department of Agricultural Botany, University College of Wales, Aberystwyth
}

Received 22.iv.75

\section{SUMMARY}

The duration of the mitotic cycle at $20^{\circ} \mathrm{C}$ was estimated in meristems of roots averaging $12 \mathrm{~mm}$ long in six species of the Triticinae. They include two diploids, Aegilops squarrosa and Triticum monococcum; two tetraploids, Triticum dicoccoides and Triticum timopheevi and two hexaploids, Triticum aestivum and Triticum spelta. The average duration of the cycle increased in the order $2 x$ (11 to 12 hours) to $4 x$ (15-16 hours) to $6 x$ (19-20 hours).

In relation to nuclear DNA amount the duration of the cycle increased by c. 0.31 hours per picogram. This rate of increase corresponds closely with that established for diploid monocotyledons by Evans et al. (1972). The mean duration of mitotic cycles also corresponds closely with that for diploid monocotyledons with the same DNA amount.

The increase in the duration of the mitotic cycle with increasing DNA is accounted for by increase in all the component phases i.e. in Division, $G 1, S$ and G2. There is some indication of a disproportionately long $G 2$ and a shorter $G 1$ in the wild, uncultivated species viz. Ae. squarrosa and the two tetraploids.

With few exceptions it appears that the duration of the mitotic cycle, as in the six species investigated here, is closely dependent upon and constrained by the nuclear DNA quantity per se.

\section{Introduction}

THERE is good evidence showing that the duration of the mitotic cycles in (root tip) meristems of species of higher plants is influenced by the nuclear DNA amount (Van't Hof and Sparrow, 1963; Van't Hof, 1965; Evans and Rees, 1971, 1972; Bennett, 1972). The duration of the cycle and of its component phases, including the synthesis $(S)$ phase, increases with increasing DNA. Evans et al. (loc. cit.) have reported some variation on this general theme. For example,

1. In diploid dicotyledons the duration of the mitotic cycle is about 4 hours longer than in diploid monocotyledons with the same DNA amount. (The rate of increase in the duration of the cycle with increasing DNA is the same in both groups, c. 0.38 hours per picogram.)

2. When the DNA content is increased by the addition of $\mathbf{B}$ chromosomes the duration of the cycle is increased to a disproportionate degree (c. 0.40 hours per picogram) in comparison with DNA increase involving chromosomes of the normal complement.

These facts tell us that while the correlation between DNA amount and the duration of the mitotic cycle is, in general, surprisingly predictable the correlation is not invariable. It is to some degree modified according to genotype, i.e. the quality as well as the quantity of nuclear DNA. 
Some authors, in contrast to the above, find no evidence of a relationship between the duration of the mitotic cycle and the nuclear DNA amount (Oehlert et al., 1962; Cameron and Stone, 1964; Graham, 1966; Troy and Wimber, 1968). There are results also from the Triticinae which conflict sharply with the correlation described above. Bayliss (1972) gives an estimate for the duration of the mitotic cycle in the hexaploid Triticum aestivum (12.5 hours) which is considerably less than that in cultivars of the tetraploid $T$. durum (Avanzi and Deri, 1969) at the same temperature. T. aestivum has, of course, about 30 per cent more nuclear DNA. Another comparably conflicting result is that of Kaltsikes (1971) to the effect that the duration of mitosis in the hexaploid Triticale is shorter than in one of its tetraploid parents, T. durum. From these results in the Triticinae two possibilities which come to mind are that hybridity and polyploidy associated with the evolution of this group or, alternatively, intensive selection for cultivation by the breeder, may have led to the disruption of the generally highly predictable relations between DNA amounts and the duration of mitotic cycles. The aim of the present work was to investigate these possibilities.

\section{Materials and methods}

(i) Material

The species used are listed in table 1. They were obtained from Professor Riley of the Plant Breeding Institute, Cambridge to whom we are very grateful. Triticum monococcum, T. spelta and T. aestivum (variety Maris Ranger) to varying degrees have been selected and bred for cultivation. The remaining species are "wild" species.

\section{(ii) Fixing, staining and autoradiography}

Seeds of each species were germinated on moist fibre glass paper at $20^{\circ} \mathrm{G} \pm 0 \cdot 2$. When the majority of seedlings had a primary root of $5-20 \mathrm{~mm}$ in length (average $12 \mathrm{~mm}$ ) these seedlings were immersed in tritiated thymidine $(2 \mu \mathrm{c} / \mathrm{ml})$ for 30 minutes, rinsed in distilled water, washed in non-radiactive thymidine $(0 \cdot 01-\mathrm{M})$ for 1 hour and returned to the fibre glass paper. Four seedlings of each species were taken at random at hourly intervals (Ae. squarrosa, T. monococcum and T. spelta) or at mainly 2-hour intervals ( $T$. dicoccoides, $T$. timopheevi and $T$. aestivum) after removal from radioactive solution. The roots were then fixed in three to one alcohol:acetic acid for 2 hours, hydrolysed for 10 minutes in $1 \mathcal{N}-\mathrm{HCl}$ at $60^{\circ} \mathrm{G}$ and then stained in Feulgen solution for 2 hours. Using previously subbed slides, root tips squashes were made and the coverslips were removed on a liquid nitrogen freezing table. The slides were passed through 70 per cent, 50 per cent and 30 per cent alcohol to distilled water. Kodak ARIO stripping film was applied and the slides, dried with silica gel, were stored in the dark at $4^{\circ} \mathrm{C}$ for two and a half weeks. The autoradiographs were developed in Kodak D19B developer for 8 minutes, washed in distilled water and fixed for 5 minutes. After washing and drying thoroughly, coverslips were applied.

(iii) Timing the mitotic cycle and its component phases

The method used was based on that of Quastler and Sherman (1959) and Wimber (1960). 


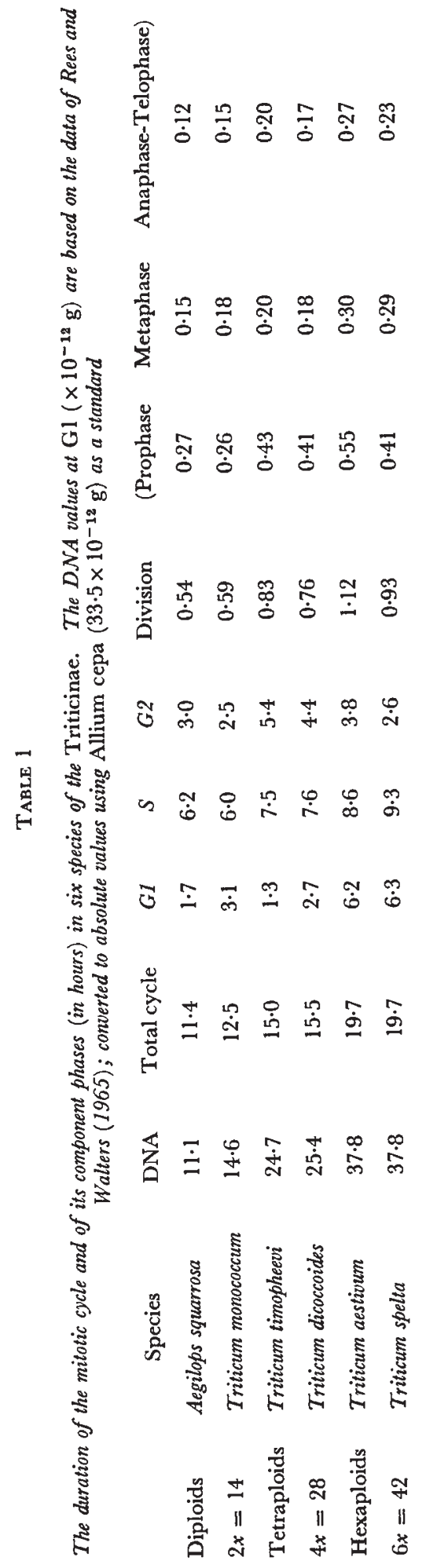


(iv) The total cycle

In fig. 1 are the percentages of labelled prophases (in $T$. monococcum and $T$. spelta), the percentages of labelled metaphases (in the remaining species) from slides fixed at intervals following labelling. The best fitting, typical two-peaked curves were derived by computer program based on the formula
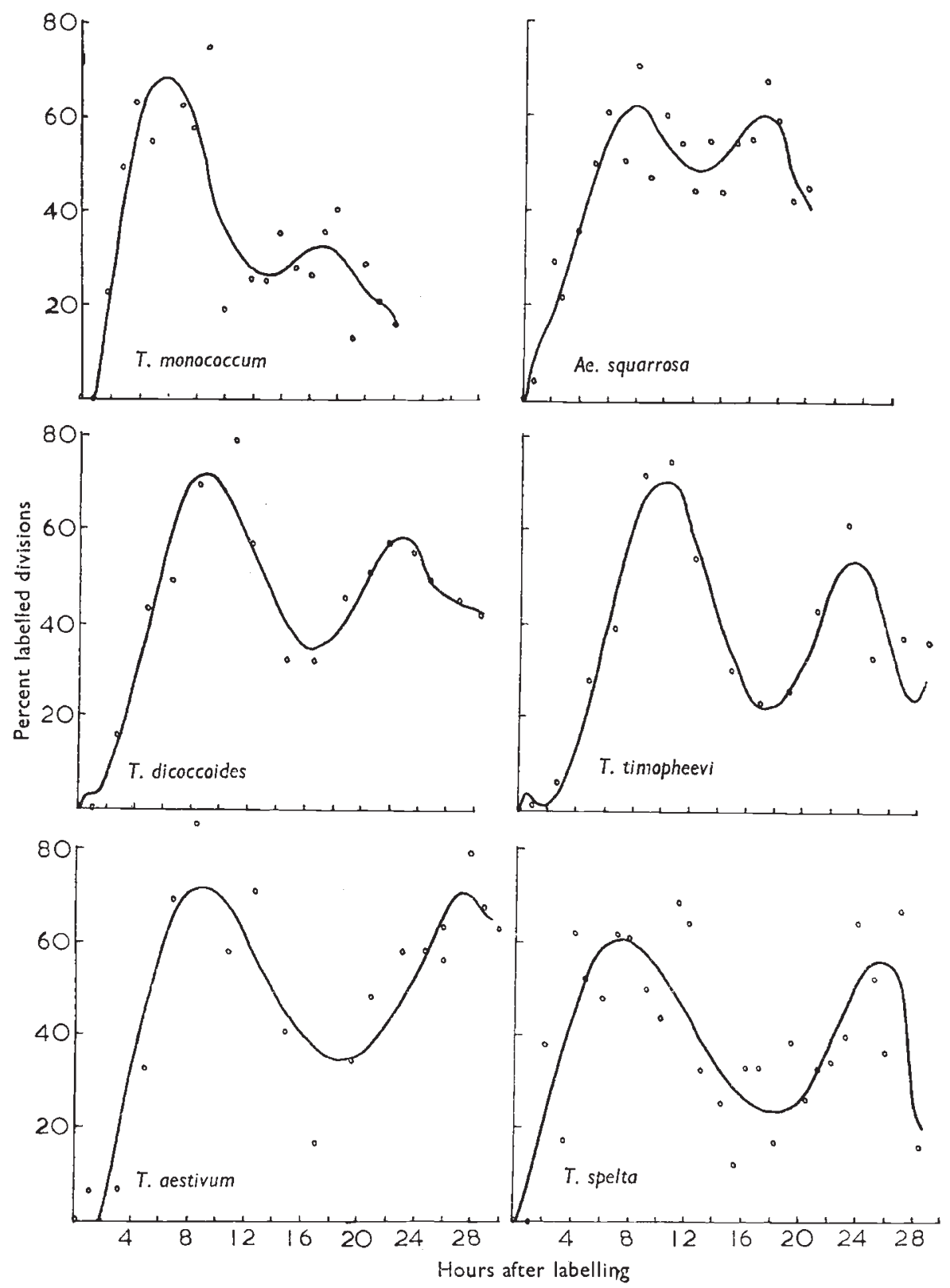

Fig. 1.-The percentages of labelled prophases in $T$. monococcum and $T$. spelta, labelled metaphases in the remainder, at intervals following labelling with tritiated thymidine. 
of orthogonal polynomials of least squares. For each species the duration of the total cycle is represented by the interval between the 50 per cent intercepts on the ascending curves of each peak.

Tritium labelled early prophases are sometimes difficult to distinguish from interphases especially in heavily labelled slides. For this reason we preferred scoring the percentage of labelled metaphases. In T.monococcum and T. spelta, however, the number of metaphases available in some slides was small and we consequently scored prophases.

It will be observed that even between peaks there is a considerable percentage of prophases or metaphases with label, ranging from about 20 to 50 per cent. We deduce from this that there is no pronounced synchrony between cells within the root meristems at this stage of development. A pronounced synchronisation would have been expected in the first few mitoses immediately following root emergence. There is evidence (Bennett and Bayliss, personal communication) indicating that the duration of the mitotic cycles and of the component phases in these earlier, more synchronised divisions are profoundly different from the divisions in older roots of the kind investigated in this experiment. This suggests that the apparently conflicting results concerning the relation between nuclear DNA amount and the duration of cell cycles from differing sources, to which we have referred earlier, may well have arisen from estimates of cycles made at different developmental stages.

\section{(v) The synthesis (S) phase}

The duration of $S$ in each case is represented by the interval between the 50 per cent intercept on the ascending part of the first peak and the 50 per cent intercept on the descending curve of that peak, minus the period of time in label i.e. 30 minutes.

\section{(vi) The division (D) phase}

The percentage of cells in division i.e. at prophase, metaphase, anaphase and telophase was estimated from 100 cells in each of 10 root tip squashes in each species. The percentage of cells, that is from prophase to telophase $x$ the duration of the total cycle in each species gives the duration of the division phase. The duration of prophase, of metaphase and of anaphasetelophase are derived in the same way i.e. percentage at prophase $\times$ the duration of the total cycle etc.

\section{(vii) The post-DNA-synthesis (G2) phase}

For $T$. monococcum and $T$. spelta, in which the percentages of labelled prophases are plotted, $G 2$ is estimated from the interval between the end of labelling ( 0 on the graphs) to the 50 per cent intercept on the first ascending curve.

It will be recalled that the curves for the other species represent percentages of labelled metaphses. For these species $G 2$ was estimated from the interval between the end of labelling to the 50 per cent intercepts on the first ascending peak minus the duration of prophase in each case. 
(viii) The pre-DNA-synthesis (G1) phase

This phase was obtained by subtraction, that is the total cycle minus $D+G 2+S$.

It is important to stress that the estimates are based on material grown in a constant environment with respect to light and temperature $\left(20^{\circ} \mathrm{C}\right)$ and from roots of the same age. Age, temperature (Bennett, et al., 1972; Brown, 1951) and other factors, including light (Halaban, 1972), mineral nutrients (Morinato and James, 1969) influence the mitotic cycles. For purposes of comparison it is clearly important, as here, to maintain a constant environment. Equally important, the results apply strictly to the conditions under which the estimates were made and equally strictly to roots at a particular phase of development.

\section{Results}

The estimates for the duration of the total mitotic cycle and of its component phases are given in table 1. Also in the table are the $G 1$ nuclear DNA amounts for the six species. It will be observed that the times for the mitotic cycles in the tetraploids are similar to those given for the tetraploid T. durum by Avanzi and Deri (1969). The duration of the cycle given for the hexaploids is more than 50 per cent greater than that quoted by Bayliss (1972).

\section{(i) The complete cycle}

The table shows the duration of the complete cycle to be much the same for species with the same chromosome numbers. It is clear, also, that the duration of the cycle increases with increasing chromosome number $(2 x-4 x-6 x)$. Increase in chromosome number is, of course, accompanied in this case with increase in nuclear DNA and in fig. 2 the duration of the complete cycle is plotted against the DNA amount for each species. The regression (duration against nuclear DNA amount) is highly significant $(P=<0.001)$. Also in the figure is the regression line calculated for diploid monocotyledons by Evans et al. (1972). It will be observed that the values for the six species investigated are distributed closely around this regression. A joint regression analysis of variance showed no significant difference between the regressions in respect either of slope or of means.

According to Evans et al. (loc. cit.) the mitotic cycles in diploid monocotyledons are of longer duration than in autopolyploid monocotyledons with similar DNA amounts. On the present evidence the same would apply to allopolyploid relative to autopolyploid monocotyledons. The conclusion must be qualified to some extent on the grounds that the data on autopolyploids presented by Evans et al. are relatively sparse and strongly influenced by the results from polyploid Hyacinthus. The latter have very high DNA amounts and the duration of the mitotic cycles in diploids (or allopolyploids) over the same range of DNA values are not available for comparison.

\section{(ii) The component phases}

Table 1 shows, in general, that increase in the duration of the complete cycle in relation to DNA content is accounted for by extension of each of the 
component phases i.e. of $S, G 2$, Division and G1. There is, however, some indication in the three wild species, Ae. squarrosa and the two tetraploids, of a disproportionately short $G 1$ and a disproportionately long $G 2$ in comparison with the three cultivated species. Whether this is of any real significance must await further comparisons between wild and "bred" forms.

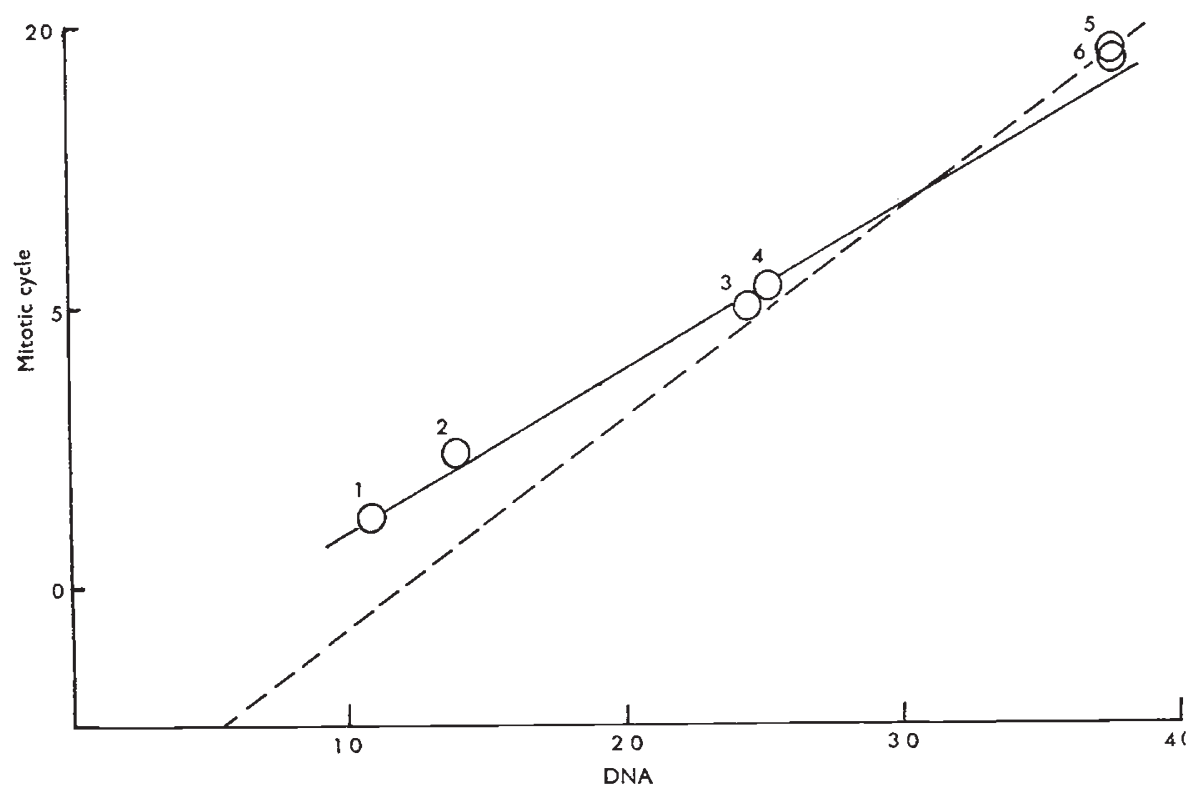

FIG. 2.-The duration of the total mitotic cycle in hours plotted against the nuclear DNA amount $\left(\times 10^{-12} \mathrm{~g}\right)$ in 1 , Aegilops squarrosa; 2, Triticum monococcum; 3, T. timopheevi; 4, $T$. dicoccoides; $5, T$. aestivum; $6, T$. spelta. The dotted line gives the expected distribution of points on the basis of a survey of diploid monocotyledons by Evans et al. (1972).

\section{Discussion}

We have shown that the correlation between the duration of the complete mitotic cycle and the nuclear DNA amount in the six Triticinae species is precisely the same as that established for diploid monocotyledons by Evans et al. (1972). Bearing in mind that the $4 x$ and $6 x$ species have a very different evolutionary history from the diploids, bearing in mind also that three of the species are wild, uncultivated forms whereas the others, to varying degrees, have been selected and bred for cultivation, the results are somewhat surprising; for two reasons. First, there is no doubting the existence of genetic mechanisms which regulate the duration of mitotic cycles in meristems during growth and development. The duration varies with the "age" of organs (e.g. of roots in Vicia, Bennett, Smith and Smith, 1972). It varies from one tissue, one organ to another. For example, the average duration of mitotic cycles in endosperm nuclei of Triticum species is reduced to a third of that in root meristem nuclei despite the fact that the former contain half as much more DNA (Bennett, personal communication). Second, the rate of growth at cell level in root as in other meristems is determined by the rate (duration) of the mitotic cycles along with the rate and extent of cell extension. On the face of it one might well have expected that selection imposed

35/3-D 
by the breeder, or in nature, to have been effective in modifying substantially, if not breaking, the dependence of mitotic cycle time upon the nuclear DNA amount. Yet the evidence, in both dicotyledons and monocotyledons, is overwhelming in demonstrating a remarkable consistency in the relationship between them. It implies a formidable constraint upon the options open for achieving change in cell growth, a constraint imposed largely by DNA amount per se.

The situation in the Triticinae is confused by the observations of Bennett (1971) that at meiosis the relation between the durations of the divisions are inversely correlated with DNA amount, so that meiosis takes longer in diploid species than in the hexaploids, the tetraploids being intermediate and also, as mentioned above, the rate of division of endosperm nuclei is largely independent of DNA amount. Indeed there is evidence to show that the first burst of mitotic cycles in seedling root tips follows the same trend as shown at meiosis i.e. longer in diploids than in hexaploids (Bennett, personal communication). What accounts for the apparent reversal of pattern during gamete formation, and seed development is not known.

There are other "deviations" reported in other families. Nagl (1974) reports that mitotic cycles in root meristems of annual species (in the Asteraceae) are of shorter duration than in perennial species with similar DNA amounts. It will be recalled that fluctuations in the relation between DNA content and mitotic cycles also applied in cases where the DNA variation was attributable to varying numbers of B chromosomes. Nevertheless, taking the evidence as a whole the dependence of the mitotic cycle time upon DNA amount is surprisingly rigid and predictable. The rigidity of the relationship is re-inforced by the present results from the wheat species investigated.

\section{REFERENGES}

AVANZI, s., AND DERI, P. L. 1969. Duration of the mitotic cycle in two cultivars of Triticum durum as measured by $\mathrm{H}^{3}$ thymidine labelling. Caryologia, 22, 187-194.

BAyliss, M. W. 1972. An analysis of meiosis in Triticum aestivum Ph.D. thesis, University of Cambridge.

BenNeTt, M. D. 1971. The duration of meiosis. Proc. Roy. Soc. Lond. B, 178, 277-299.

BENNETT, M. D. 1972. Nuclear DNA content and minimum generation time in herbaceous plants. Proc. Roy. Soc., B, 181, 109-135.

BENNETT, M. D., SMITH, J. B., AND SMTTH, D. 1972. The mitotic cycle duration in Vicia faba root-tip meristems with different sized chromosomes. Caryologia, 25, 445-453.

BROWN, R. 1951. The effects of temperature on the division of different stages of cell division in the root tip. 7. Expl. Bot., 2, 96-110.

CAMERON, I. L., AND STONE, G. E. 1964. Relation between the amount of DNA per cell and the duration of $D \mathcal{N} A$ synthesis in three strains of Tetrahymena pyriformis. Expl. Cell Res., $36,510-514$.

EVANS, G. M., AND REES, H. 1971. Mitotic cycles in dicotyledons and monocotyledons. Nature, 223, 350-351.

EVANS, G. M., REES, H., SNELL, C. L., AND SUN, s. 1972. The relationship between nuclear DNA amount and the duration of the mitotic cycle. Chromosomes Today (ed. Darlington, C. D. and Lewis, K. R.) 3, 24-31.

GRAHAM, C. F. 1966. The effect of cell size and DNA content on the cellular regulation of DNA synthesis in haploid and diploid embryos. Expl. Cell Res., 43, 13-19.

HALABAN, R. 1972. Mitotic index and cell cycle of Lemna perpusilla under different photoperiods. Plant Physiol., 50, 308-310.

KALTSIKEs, P. J. 1971. The mitotic cycle in an amphiploid (Triticale) and its parental species. Can. J. Genet. Cytol., 13, 656-662. 
MORINATO, H., AND JAMES, T. w. 1969. Sulphate controlled synchrony of Astasia lorga. Exp. Cell. Res., 58, 195-200.

NAGL, w. 1974. Mitotic cycle time in perennial and annual plants with various amounts of DNA and heterochromatin. Dev. Biol., 34, 342-346.

OEHLERT, W., SEEMAYER, N., AND LAUF, P. 1962. Autoradiographic research on the generation cycle of the Ehrlich ascites carcinoma in the white mouse. Beitr. path. Anat., 127, 63-68.

QUASTLER, H., AND SHERMAN, F. G. 1959. Cell population kinetics in the intestinal epithelium of mouse. Exp. Cell. Res., 17, 420-438.

REES, H., AND WALTERS, M. R. 1965. Nuclear DNA and the evolution of wheat. Heredity, 20, 73-82.

TROY, M. R., AND WIMBER, D. E. 1968. Evidence for a constancy of the DNA synthetic period between diploid-polyploid groups in plants. Expl. Cell Res., 53, 145-154.

VAN'T HOF, J. 1965. Relationships between mitotic cycle duration, S. period duration and the average rate of DNA synthesis in the root meristem cells of several plants. Expl. Cell Res., 39, 48-58.

VAN'T HOF, J., AND SPARROW, A. H. 1963. A relationship between $D \mathcal{N} A$ content, nuclear volume and minimum mitotic cycle time. Proc. Nat. Acad. Sci., 49, 897-902.

WIMBER, D. E. 1960. Duration of the nuclear cycle in Tradescantia paludesa root tips as measured with $H^{3}$ thymidine. Amer. F. Bot., 47, 828-834. 